\section{Ubiquitin tug-O-war}

$\mathrm{U}$ biquitin (Ub) conjugation regulates numerous cellular processes, yet past biochemistry experiments suggested that although cells have a lot of Ub, little of it is free. On page 19, Dantuma et al. show that recruiting Ub to one site pulls it off another and, thus, Ub may link otherwise distinct processes, such as protein degradation and gene transcription.

GFP-Ub was evenly distributed in the nucleoplasm and appeared in many small foci in the cytoplasm. During photobleaching experiments, only a small fraction of the Ub diffused freely between the cytosol and the nucleus, indicating that most of it was conjugated to larger molecules. Moreover, about $70 \%$ of the Ub in the nucleus was immobile, consistent with its conjugation to histone $\mathrm{H} 2 \mathrm{~A}$.

\section{Endosome signaling}

W ingless $(\mathrm{Wg})$ signals mostly from the endosome not from the plasma membrane, according to Seto and Bellen (page 95).

The Wnt/Wg pathway controls numerous steps in development and is tightly regulated. The receptor and coreceptor for $\mathrm{Wg}$ sit on the cell surface where they can bind extracellular ligand, but signaling relies on the presence of downstream proteins.

One possible site for uniting receptors and signaling proteins is on endosomes-after endocytosis of the Wg-receptor complex, but before lysosomal degradation. To test this possibility, Seto and Bellen asked what happened to $\mathrm{Wg}$ signaling when they used mutations in key genes to block individual vesicle trafficking steps between the cell surface and the lysosome.

When endocytosis was inhibited, a large excess of $\mathrm{Wg}$ accumulated on the cell surface but little downstream signaling occurred. Similarly, when endocytosis was allowed, but vesicle fusion to the endosome was hindered, little signaling occurred. By contrast, when the Wg-receptor complex made it to the endosome but was barred from moving into multivesicular bodies and lysosomes, signaling was robust and persistent. Overexpression of $\mathrm{Hrs}$, a protein that promotes multivesicular body formation, decreased $\mathrm{Wg}$ signaling.

Endosome-based signaling may provide precise temporal regulation because the cell can control the duration a protein complex stays in the endosome. That regulatory control may be important in the Wg pathway, as some effector proteins are known to need more or less ligand for activation. JCB
When GFP-Ub-expressing cells were exposed to proteotoxic stress, $\mathrm{Ub}$ translocated rapidly from the nucleus to the cytosol, and much of the cytoplasmic Ub moved at a rate consistent with conjugation to proteasome substrates. Simultaneously, $\mathrm{H} 2 \mathrm{~A}$ became increasingly deubiquitylated, causing chromatin condensation.

One possibility is that the cell simply cannot make enough Ub to satisfy its needs and must poach one Ub store in favor of another. However, the team hypothesizes that the cell relies on the limited pool of Ub to provide cross-talk between distant processes. For example, their preliminary data suggest that transcription levels may change in response to an accumulation of misfolded proteins. JCB
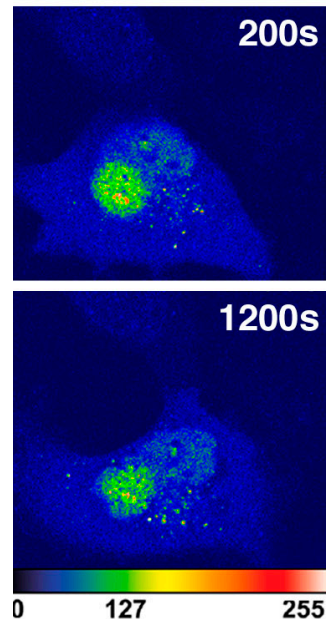

Nuclear ubiquitin is poached for cytoplasmic protein degradation.

\title{
Unscrambling secretion
}

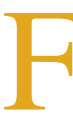

lies lacking both scramblase genes show normal phospholipid distribution and scrambling in response to physiological stimuli, report Acharya et al. on page 69. The mutant animals, however, have increased synaptic vesicle activity at the neuromuscular junction, leading the team to hypothesize that scramblases may modulate secretory activity.

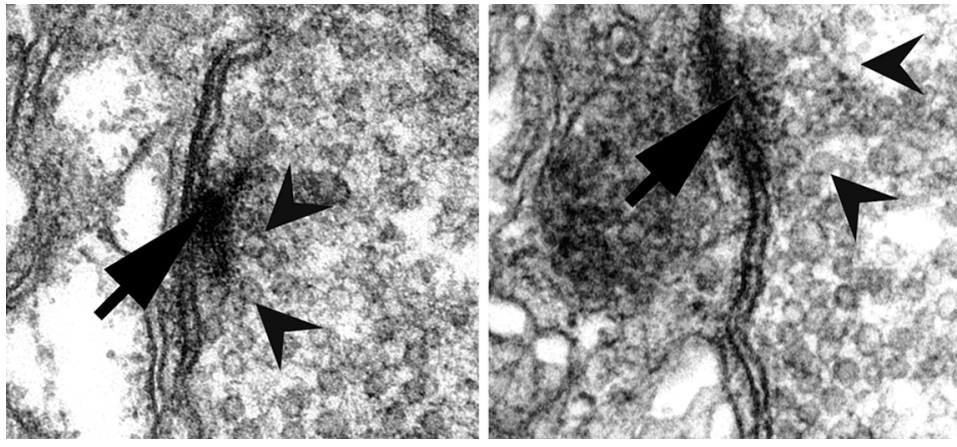

Flies without scramblases (right) have extra synaptic vesicles.

Scramblases were initially identified in human blood cells through a search for proteins that could transfer phospholipids from one membrane leaflet to the other. Yet mice lacking two of the four mouse scramblase genes showed normal phospholipid scrambling. Therefore, either the other two homologues compensated for scrambling function or the protein family has a previously unidentified function.

To find out, Acharya et al. generated flies lacking both of the Drosophila scramblase genes. During apoptosis, phosphatidylserine moved from the inner leaflet to the outer one as expected, indicating that the scrambling function remained intact in cells lacking either or both scramblases. Scrambling was also normal during phagocytosis in bacteria-infected animals.

The double-mutant flies were, however, jumpy in their vials, a phenotype previously associated with synaptic function defects. When the team examined the structure and function of the neuromuscular junction, they found an excess of both synaptic vesicles trafficking and neurotransmitter release, relative to wild type. This is consistent with previous studies linking scramblase proteins to modulation of secretory pathways. JCB 